\title{
Lung Segmentation on Postero-anterior Digital Chest Radiographs Using Active Contours
}

\author{
Isaac Iglesias ${ }^{1}$, Pablo G. Tahoces ${ }^{2}$, Miguel Souto ${ }^{3}$, Anxo Martínez de Alegría ${ }^{3}$, \\ María J. Lado ${ }^{4}$, and Juan J. Vidal ${ }^{3}$ \\ 1 Laboratory for Radiologic Image Research, University of Santiago, 15782 Santiago \\ isaacigl@usc.es \\ 2 Department of Electronics and Computer Science. University of Santiago \\ Campus Sur s/n, 15782 Santiago \\ 3 Department of Radiology \\ Complejo Hospitalario Universitario de Santiago (CHUS) \\ University of Santiago, 15782 Santiago \\ 4 Department of Computer Science, University of Vigo \\ Campus Lagoas-Marcosende, 36200 Vigo
}

\begin{abstract}
A computerized pulmonary segmentation based on the detection of oriented edges was performed in postero-anterior (PA) digital radiography (DR) images. To further improve detection of lung contours, a method based on the use of active contours models was developed. First, the technique calculates a set of reference lines to determine the relative position of the lungs in the image. Then, vertical and horizontal rectangular regions of interest (ROIs) are studied to identify the preliminary edge. These points are an approximation to the lung edges that are adjusted using the active contours models. We studied the influence of the different parameters of the active contours on the final result over 30 DR images. Results prove that the active contour models, with selected parameters, can be used to improve the results of a given segmentation scheme.
\end{abstract}

\section{Introduction}

Digital radiography (DR) is becoming the standard for pulmonary imaging [1] [2]. DR provides high spatial and contrast resolution for the lung parenchyma. DR also opened the possibilities for computer-aided diagnosis (CAD) applications in chest imaging, such as pulmonary nodules detection [2] [3], characterization of interstitial disease [4], measurement of pulmonary volume [5], and detection of cardiomegaly [6] and pneumotoraces [7]. A survey of the CAD in the chest radiography can be found in [8].

Lung segmentation is a necessary prerequisite for all of these quantitative analysis applications. Several investigators have developed techniques for CAD segmentation of pulmonary DR images. Xu and Doi [9] have determined the rib cage boundary using a set of ROIs, which are selected over the maxima and minima of the gray-level values on horizontal profiles. Duryea et al. [10] searched the 
lung contour studying the difference between the consecutive maxima and minima of horizontal profiles. Armato et al. employed an iterative global gray-level thresholding and a local gray-level threshold analysis with the resulting image to determine the lung edge in PA [11] and lateral [12] chest radiographs; Carrascal et al. [5] determined a group of reference lines, defined a set of rectangular ROIs and identified the pulmonary border using edge enhancement and thresholding techniques. Vittitoe et al. [13] employed Markov random field modeling for identifying lung regions. Tsujii et al. [14] classified the pixels of the DR using an adaptative-sized hybrid neural network. Van Ginneken et al. [15] developed and compared several segmentation techniques: a matching approach, pixel classifiers based on several combinations of features, a rule-based scheme that detects lung contours using a general framework for the detection of oriented edges and ridges in images, and a hybrid scheme. Later, an active shape model was used by van Ginneken et al. [16] to obtain the segmentation.

Investigators use different algorithms to obtain the segmentation. Of interest is that there is a common step to all of them: it consists on the adjustment of an initial contour in order to correct, complete or smooth it. We believe that the active contour models (snakes) can be very helpful to perform this task. This technique was previously used in chest radiography by Yue et al. [17] to the detection of the rib borders. We propose an automatic lung segmentation scheme that calculates a set of reference lines to determine the relative position of the lungs in the image. Using these lines, a collection of rectangular ROIs to cover the pulmonary border is sequentially defined. To obtain the points of the border, the averaged horizontal or vertical profile in each ROI was analyzed. Finally, the algorithm fits the set of points using an active contour model. The influence of different parameters of the active contour models, such as the relative weights of the three energies (continuity, curvature and image energy) was studied.

\section{Materials}

A total of 30 PA chest radiographs were employed to develop the method. DR was performed using a Siemens Thorax FD (Siemens AG, Munich, Germany) with a matrix of $3 \mathrm{~K} \times 3 \mathrm{~K}$ (pixel size of $143 \mu \mathrm{m}$ ) and 4096 gray level resolution in DICOM format. No distinction was made between normal and pathological cases. The computer programs were written in $\mathrm{C}++$ programming language on Solaris 2.8 operating system. CNT (Mallinckrodt Institute of Radiology, Washington University School of Medicine) libraries have been used for development of DICOM utilities. A Sun Ultra80 Workstation (Sun Microsystems, Inc., Mountain View, CA) was used for all the calculations.

\section{Methods}

To develop the computerized system to automatically extract the lung contour, we have followed three steps: a) calculation of the reference lines; b) calculation of the points near the lung edge; and c) correction of the rough border using a snake. 


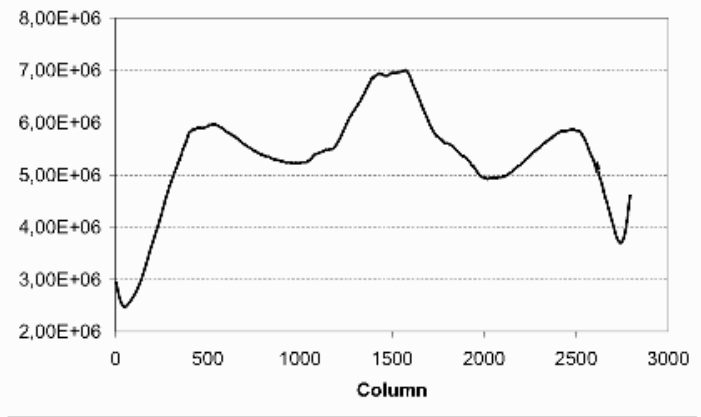

(a)

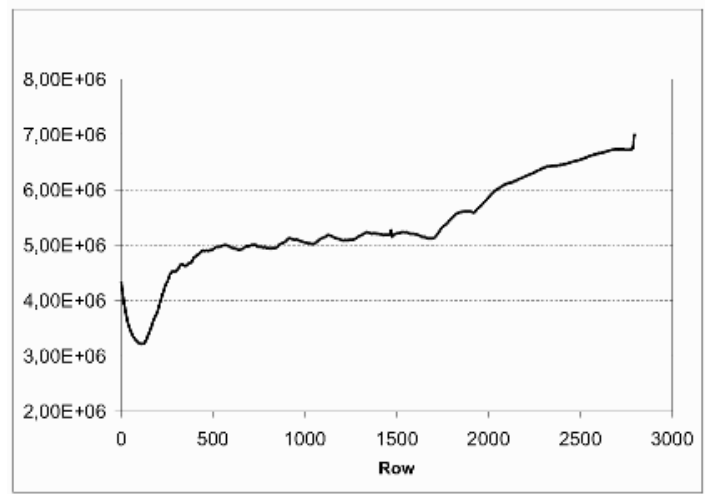

(b)

Fig. 1. Horizontal 1(a) and vertical signature 1(b)

\subsection{Reference Lines}

We used the definition of horizontal and vertical thoracic signature (equations 1 and 2) suggested by Meyers et al. [18]:

$$
\begin{aligned}
S_{h o r}(j) & =\sum_{i=0}^{N_{f i l}} I[i, j] \\
S_{\text {ver }}(i) & =\sum_{j=0}^{N_{c o l}} I[i, j]
\end{aligned}
$$

and that are represented in the figures $1(\mathrm{a})$ and $1(\mathrm{~b})$. The midline $C_{\text {mid }}$ (1 in figure 2) of the thoracic cage was determined by searching the central maximum of the horizontal signature of the image (figure 1(a)). The upper and lower limits of the rib cage were obtained from the vertical signature (figure $1(\mathrm{~b})$ ), the maximum in the first third of the derivative being the upper limit $R_{u p}(2)$, and 
the lower limit being the maximum of the last half of the derivative. Since the lower limit of the left lung can be very different of the lower limit of the right lung we calculated the vertical signature of each hemithorax and we obtained two lower limits, $R_{\text {low }}^{\text {right }}(3)$ and $R_{\text {low }}^{\text {left }}(4)$. The lateral limits of the lungs were calculated as follows: we calculated for each hemithorax an averaged horizontal profile of the $\frac{R_{\text {up }}-R_{\text {low }}}{3}$ rows over $R_{\text {low }}$. The external lateral limit of the right lung $C_{\text {ext }}^{\text {rigth }}(5)$ was the first maximum of the profile an the internal limit $C_{\text {int }}^{\text {right }}$ (7) was the maximum of the derivative close to $C_{\text {mid }}$. For the left lung, $C_{\text {ext }}^{\text {left }}(6)$ was the last maximum and $C_{i n t}^{\text {left }}(8)$ was the minimum of the derivative close to $C_{m i d}$. In the figure 2 the reference lines are shown.

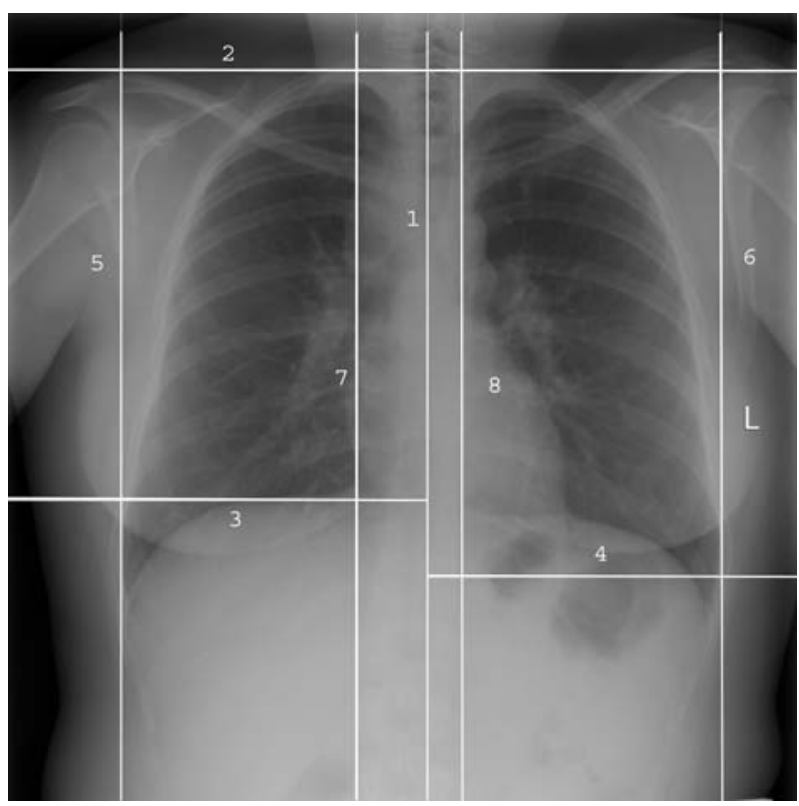

Fig. 2. Reference lines

\subsection{Calculation of the Initial Points}

Lung contour was divided into four zones: apex, mediastinum, diaphragm and rib cage border, that were calculated separately. The edge points were calculated by using a set of rectangular ROIs oriented either vertically (in the apex and the diaphragm) or horizontally (in the mediastinum and rib border). The position of these ROIs was determined by using the reference lines. In the apex, the ROIs had 30 columns width, and were located under $R_{\text {up }}$. The ROIs of the diaphragm were of the same size being calculated sequentially between $C_{\text {int }}$ and $C_{\text {ext }}$, the first was calculated around $R_{\text {low }}$ and the position of the edge point then calculated determined the position of the next ROI. The first and the last points of the diaphragm determined the position of the first ROIs of the rib cage border and 
the mediastinum, the remainder are calculated from the edge points obtained. In order to calculate the edge, the points of each ROI were averaged to obtain an horizontal or vertical average profile. To reduce the number of candidates, every point of the profile was averaged with its 30 neighbors, taking into account that we were searching a large structure such as the lung edge. After this, depending on the type of border that we were searching, the minima or the maxima of the first derivative were calculated. If there were more than one candidate, the difference between the density of gray levels of the 50 neighbors after and before the point was calculated and the one that had the greater difference in absolute value was chosen.

\subsection{Correction of the Border Using a Snake}

With the method described above we calculated an outline of the lung edge. The points did not join smoothly and any of the points did not correspond with the true lung border, because of this the border needed to be adjust. To do this we used the energy-minimizing curves known as snakes introduced by Kass et al. [19] for detecting lines and curves in an image. The snake is an active contour model which constantly minimizes an energy functional that consists on both the internal and external energy. The internal energy serves to impose continuity and smoothness constraint on the shape of the snake while the external energy tends to pull the snake toward salient image features such as image edges. Representing the snake by a parametric curve $v(s)=[x(s), y(s)]$, the energy functional can be written as:

$$
\mathcal{E}_{\text {snake }} \equiv \int_{0}^{1} E_{\text {snake }}(v(s)) d s=\int_{0}^{1}\left[E_{\text {int }}(v(s))+E_{\text {ext }}(v(s))\right] d s
$$

This expression must be discretized for using it in a digital image. Then the curve $v(s) \approx v_{i}=\left(x_{i}, y_{i}\right)$, where $0 \leq i<N$, being $N$ the number of points of the snake. In this way, the expression 3 can be written as:

$$
\mathcal{E}_{\text {snake }}=\sum_{i=0}^{N}\left(E_{\text {int }}(i)+E_{\text {ext }}(i)\right)
$$

To minimize this expression Kass et al. [19] used techniques of variational calculus, Amini et al. [20] pointed out some problems with this approach and proposed an algorithm using dynamic programming. Since this method is slow we decided to use the greedy algorithm proposed by Williams et al. [21] that defines the internal and external energies as follows:

$$
\mathcal{E}_{\text {snake }}=\sum_{i=0}^{N}\left[\alpha(i)\left(\bar{d}-\left|v_{i}-v_{i-1}\right|\right)+\beta(i)\left|v_{i-1}-2 v_{i}+v_{i+1}\right|+\gamma(i) E_{\text {image }}(i)\right]
$$

where $\bar{d}$ is the average distance between the points of the snake, $E_{\text {image }}$ is a function of the point $i$ of the image and the parameters $\alpha, \beta$ and $\gamma$ govern the 


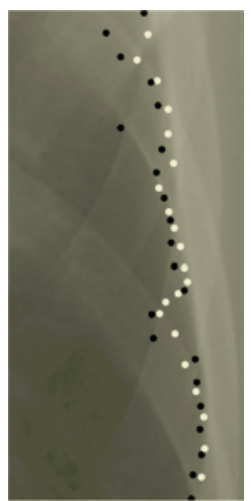

(a)

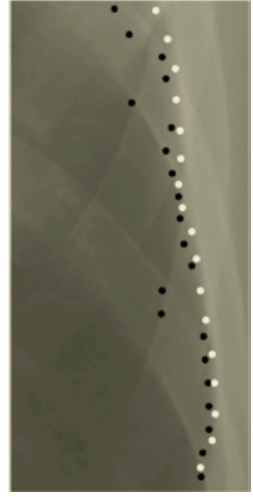

(b)

Fig. 3. Fitted rib cage border: using a gradient filter (3(a)) and a Sobel filter (3(b)) as image energy. The black dots are the initial and the white the fitted contour

relative weight of the energies. The evolution of the snake depends on the election of the function $E_{\text {image }}(i)$ that allows for determining the type of features of the image that attract the snake and the parameters that allows to determine the shape of the snake. Since each zone of the lung border has different characteristics, a snake with several values to the parameters and $E_{\text {image }}(i)$ was created, depending on the position of the point. Then four sets of parameters and four $E_{\text {image }}(i)$ (apex, mediastinum, diaphragm and rib cage border) were searched.

\section{Results and Discussion}

We calculated the initial border in $30 \mathrm{PA}$ chest images, in order to obtain the necessary features of the snake that best fitted the lung contour. The influence of different image energies (the gradient and the Sobel filters to detect horizontal and vertical edges) was studied using standard parameters. The final border was calculated and, for each zone the energy that obtained the best result was selected. In the active contour schemes the use of $E_{\text {image }}=-\operatorname{grad}[I(x, y)]$ is usual. However, in the thorax DR images this energy did not obtain the best result because there are many edges in the image, some of them become more pronounced than the lung contour. The best example of this are the ribs, when the initial contour was adjusted with $E_{\text {image }}=-\operatorname{grad}[I(x, y)]$, the snake in the rib border tended to move closer the ribs. This effect is shown in figure 3 . To solve this problem, we used the knowledge about the direction of the edges that we were searching. Each zone in the lung have different characteristics, the apex and the diaphragm are mainly horizontal edges and the mediastinum and rib border are vertical. Then we used the Sobel filters $\left(S_{x}[I(x, y)]\right.$ and $\left.S_{y}[I(x, y)]\right)$ as the $E_{\text {image. }}$ In the figure 3 is shown how this election changed the final result. With this energies, the influence of the parameters was analysed. In order to reduce the number of possibilities we only used three values $(0,5,1$ and 1,5$)$ 


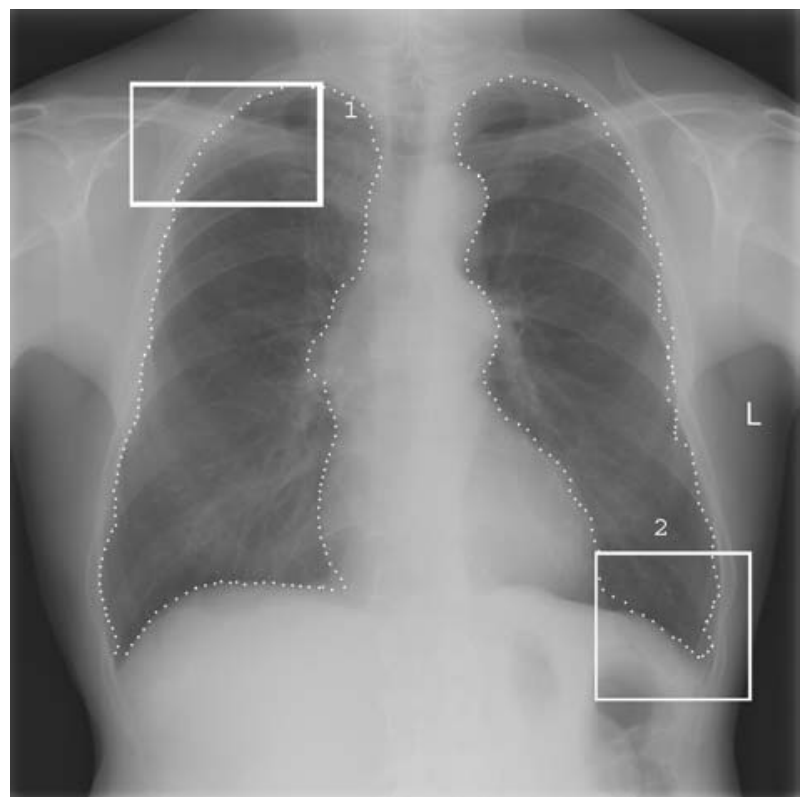

(a)

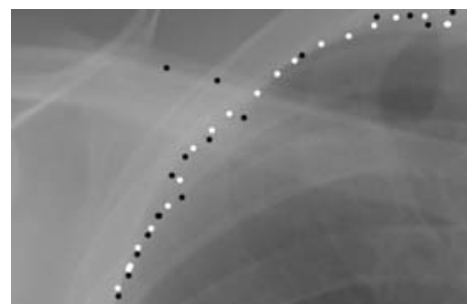

(b)

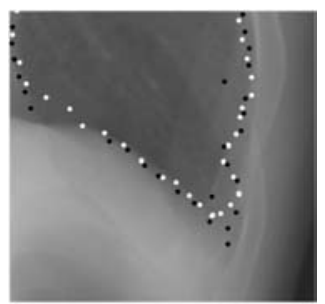

(c)

Fig. 4. 4(a) Resulting lung border. Comparison of the initial (black dots) and final contour (white dots): 4(b) in the clavicle region (1); 4(c) in the cardiophrenic and costophrenic angles (2)

for the parameters $\alpha, \beta$ and $\gamma$. Then, there were 27 possible combinations of parameters for each zone. Since the features of the initial border were known, not all of this possibilities were used in the posterior analysis. For example, it was known that the initial contour was very irregular. However, the lung contour must be rounded and as a result the $\beta$ parameter should be 1 or 1,5 to eliminate the corners. In the same way $\gamma$ should not be very small for not loosing the image information. Then the combinations for which $\gamma$ was less than the other parameters were eliminated.

In the first stage, we obtained the final border after being fitted using the remainder combinations of parameters. An experienced radiologist selected the two or three sets of parameters that best fitted in each region. Once the sets of 
parameters were selected the fit of the initial border was repeated, but employing different values for the parameters of each region. In the final step, a radiologist evaluated the accuracy of each calculated contour and the parameters that obtained the best result was chosen. In figure 4(a) an example of the final lung contour obtained is shown. The $\beta$ parameter is always high in order to obtain a smoothness effect, eliminating the irregularities in the contour. The $\alpha$ parameter tends to approximate the points of the snake. It helps in the task of eliminating the irregularities and is fundamental to correct the wrong edge points (example in fig. 4(b). As the first contour is often wrong in the ribs and the clavicle (fig. 3 and $4(\mathrm{~b})$ ) it was necessary to increase the value of this parameter in this region. On the other hand, if $\alpha$ and $\beta$ are high, the snake tends to eliminate the true lung corners, such as the cardiophrenic and costophrenic angles, specially when their edges are fuzzy. To reduce this effect, the parameters $\alpha$ and $\beta$ for the diaphragmatic region are low compared to the other regions. With a correct choice of the parameters, this effect was reduced but not eliminated as we have shown in the fig. 4(c). To improve the detection, an alternative method to carefully detect these angles, and a snake with an attractive term of energy [19] could be used in these regions.

Finally, the best results were obtained with $(\alpha=1, \beta=1.5, \gamma=1)$ for the rib border, $(\alpha=0.5, \beta=1.5, \gamma=1.5)$ for the apex, $(\alpha=0.5, \beta=1,5, \gamma=1)$ for the mediastinum and ( $\alpha=0.5, \beta=1, \gamma=1.5$ ) for the diaphragm.

These results demonstrate that a snake with the appropriate features can be used to correct, smooth and complete the results of a given segmentation scheme.

\section{References}

1. M. Souto, K. S. Malagari, D. Tucker, P. G. Tahoces, J. Correa, V. S. Benakis, C. Roussos, K. A. Strigaris, J. J. Vidal, G. T. Barnes, and R. G. Fraser. Digital radiography of the chest: state of the art. Eur. Radiol., 4:281-297, 1994.

2. J. Correa, M. Souto, P. G. Tahoces, K. S. Malagari, D. Tucker, J. J. Larkin, J. Kuhlman, G. T. Barnes, E. Zerhouni, R. G. Fraser, and J. J. Vidal. Digital chest radiography: comparision of unprocessed and processed images in the detection of solitary pulmonary nodules. Radiology, 195:253-258, 1995.

3. Maryellen L. Giger, Kunio Doi, and Heber MacMahon. Image feature analysis and computer-aided diagnosis in digital radiography. automated detection of nodules in peripheral lung fields. Medical Physics, 15:158-166, 1988.

4. S. Katsuragawa, Kunio Doi, and Heber MacMahon. Image feature analysis and computer-aided diagnosis in digital radiography: detection and characterization of interstitial lung disease in digital chest radiographs. Medical Physics, 15:311-319, 1988.

5. Francisco M. Carrascal, José M. Carreira, Miguel Souto, Pablo G. Tahoces, Lorenzo Gómez, and Juan J. Vidal. Automatic calculation of total lung capacity from automatically traced lung boundaries in postero-anteior and lateral digital chest radiographs. Medical Physics, 25 (7):1118-1131, July 1998.

6. Nobuyuki Nakamori, Kunio Doi, Victoria Sabeti, and Heber MacMahon. Image feature analysis and computer-aided diagnosis in digital radiography: Automated analysis of sizes of heart and lung in chest images. Medical Physics, 17 (3):342-350, May/Jun 1990. 
7. Shigeru Sanada, Kunio Doi, and Heber MacMahon. Image feature analysis and computer-aided diagnosis in digital radiography: Automated delineation of posterior ribs in chest images. Medical Physics, 18 (5):964-971, Sep/Oct 1991.

8. Bram van Ginneken, Bart M. ter Haar Romeny, and Max A. Viergever. Computeraided diagnosis in chest radiography: A survey. IEEE Trans. Med. Imag., 20 (12):1228-1241, December 2001.

9. Xin-Wei Xu and Kunio Doi. Image feature analisys for computer aided diagnosis: Accurate determination of ribcage boundary in chest radiographs. Medical Physics, 22 (5):617 - 626, May 1995.

10. Jeff Duryea and John M.Boone. A fully automated algorithm for the segmentation of lung fields on digital chest radiographics images. Medical Physics, 22 (2):183191, February 1995.

11. Samuel G. Armato III, Maryellen L. Giger, and Heber MacMahon. Automated lung segmentation in digitized posteroanterior chest radiographs. Acad. Radiol., 5:245 - 255, 1998.

12. Samuel G. Armato III, Maryellen L. Giger, Kazuto Ashizawa, and Heber MacMahon. Automated lung segmentation in digital lateral chest radiographs. Medical Physics, 25 (8):1507 - 1520, August 1998.

13. Neal F. Vittitoe, Rene Vargas-Voracek, and Carey E. Floyd, Jr. Identification of lung regions in chest radiographs using markov random field modeling. Medical Physics, 25 (6):976 - 985, June 1998.

14. Osamu Tsujii, Matthew T. Freedman, and Seong K. Mun. Automated segmentation of anatomic regions in chest radiographs using an adaptative-sized hybrid neural network. Medical Physics, 25 (6):998 - 1007, June 1998.

15. Bram van Ginneken and Bart M. ter Haar Romeny. Automatic segmentation of lung fields in chest radiographs. Medical Physics, 27 (10):2445-2455, October 2000.

16. Bram van Ginneken, Alejandro F. Frangi, Joes J. Staal, Bart M. ter Haar Romeny, and Max A. Viergever. Active shape model segmentation with optimal features. IEEE Trans. Med. Imag., 21 (8):924-933, August 2002.

17. Zhanjun Yue, Ardeshir Goshtasby, and Laurens V. Ackerman. Automatic detection of rib borders in chest radiographs. IEEE Trans. Med. Imaging, 14 (3):525 - 536, September 1995.

18. P. H. Meyers, H. C. Nice, C. M.and Becker, N. J. Nettleton, J. W. Sweeney, and G. R. Meckstroht. Automated computer analysis of radiolographic images. Radiology, 13:1029-1034, 1964.

19. M. Kass, A. Witkin, and D. Terzopoulos. Snakes: Active contour models. International journal of computer vision, 4:321-331, 1988.

20. A. A. Amini, T. E. Weymouth, and R. C. Jain. Using dynamic programming for solving variational problems in vision. IEEE Trans. Pattern Anal. Machine Intell., $12(9): 855-867,1990$.

21. D. J. Williams and M. Shah. A fast algorithm for active contour and curvature estimation. CVGIP: Image Understanding, 55 (1):14 - 26, 1992. 